\title{
Computational Analysis of the Capacitance by the Boundary Element Method
}

\author{
Twaibu Semwogerere ${ }^{1 *}$, Richard Awich ${ }^{2}$, Hasifa Nampala ${ }^{3}$ \\ ${ }^{1}$ Faculty of Engineering, Busitema University, Tororo, Uganda. \\ 2 Faculty of Science Education, Busitema University, Tororo. \\ ${ }^{3}$ Department of Mathematics, Kyambogo University, Kampala. \\ * Corresponding author. Tel.: +256 772353 598; email: semwogereretwaibu@yahoo.co.uk \\ Manuscript submitted February, 17, 2018 and accepted on May 15, 2018. \\ doi: 10.17706/ijapm.2018.8.4.79-89
}

\begin{abstract}
The boundary element method (BEM) has had an advantage of discretizing the boundary over the finite element method and the finite difference method for so long. The curved and straight elements are crucial for the discretization process. The BEM has been of fundamental importance in as far as solving a two -dimensional Laplace problem is concerned. The study aimed at using the BEM to compute the capacitance as compared to the ordinary physics methods. The BEM and its applications were reviewed in relation to curved and straight elements. The Dirac-Delta and the Green's functions were behind the use of the BEM. A model problem was tested for analysis of the BEM with curved elements in relation to the computation of the capacitance. The MATLAB programs (Beextcp.m, Beextc. $m$, Beextcp5.m, ansep.m, eltsp.m, anse1.m,Eltsp5.m, Mant2.m, and Trapez.m) and subprograms were used among others in solving the problem and also in the analysis. Findings involved the computation of the matrix terms for curved elements in relation to the cylinder curvatures. Findings also showed and compared three methods of computing the capacitance in a model problem from electrostatics. The concentric cylinder considered in this study had different shape plates where the BEM showed a fundamental advantageous stage of the BEM in calculating the capacitance for various curvature plate shapes than any other numerical method by simply increasing on the number of elements. The BEM avoids assumptions about the inner plate and treating the situation as a connected domain.
\end{abstract}

Key words: Boundary element method, capacitance, curved, elements, formulation.

\section{Introduction}

There are several mathematical problems that arise in our day-to-day lives. Some of these problems are the boundary problems [1], [2]. Such problems can be solved by several analytical and numerical methods which include the BEM, among others. The BEM simply requires the discretization of the boundary than the whole domain. It should be noted that the BEM is more recent than the other two and has several advantages over the others that include among others: Discretizing at infinity [3], and its application to solving acoustic and general engineering science problems [2].

For instance, the boundary problems are a common phenomenon in mathematics [1]. An example of the boundary value problem is:

Let $E$ be an open domain in $R^{N}$ with boundary $S$, 
where $g(x)$ is the Dirichlet data [4].

$$
\begin{gathered}
-\nabla^{2} u(x)=0, x \in E \\
u(x)=g(x) \text { on } S
\end{gathered}
$$

Such a problem can be solved by several analytical and numerical methods which include the BEM amongst others. The FEM helps in the understanding and the use of the BEM [4], [5]. It should be noted that the BEM is more recent than the other two and has several advantages over the others that include among others: Discretizing at infinity [4], [6]-[8], its application to solving acoustic problems, in simplifying general engineering science problems, and the interior or exterior Laplace problems.

The manuscript mainly entails the use of the BEM on the curved elements in evaluating the capacitance of concentric cylinders. This requires preliminary studies of the Dirac-delta distributions, the Green's functions, and the divergence theorem. These were used in this study at particular intervals though their details were not considered. Similarly, the Laplace equation, electro-statics (Capacitance) and the BEM were briefly reviewed.

\subsection{The Laplace Equation}

This equation is considered in electro-statistics, and it is also used in incompressible fluid flow and for the heat conduction in the steady state [8]. For a Laplace equation, the solutions within a given region say $R$, can be found given the specification of the potential or field around the boundary. The Laplace equation [4] was defined as follows for the space and can be reduced to that of a plane:

$$
\nabla^{2} u=\frac{\partial^{2}}{\partial x^{2}}+\frac{\partial^{2}}{\partial y^{2}}+\frac{\partial^{2}}{\partial z^{2}}=0
$$

Solutions of Laplace's equation are called harmonic functions like that in equation (2) [4], [9]. The Laplace's boundary integral form required us to transform the PDE only unlike the FEM. A function $\boldsymbol{u}$ was considered to be satisfying equation (1) to give the integral formulation in equation (3) [4]:

$$
\int_{S}\left[G(s ; x) \frac{\partial u}{\partial n}(s)-u(s) \frac{\partial G}{\partial n}(s)\right] d s=\left\{\begin{array}{c}
u(x), \xi \in D \\
0.5 u(x), \xi \in S \\
0, \xi \in E
\end{array}\right.
$$

where $G$ is the Green's function, $D$ is the interior domain, $S$ is the boundary and $E$ is the exterior region. This accomplishes the first stage of the BEM [1].

\subsection{The Boundary Element Method (BEM)}

\subsubsection{Introduction}

The FEM and FDM [2] and [10] are less recent than the BEM in their application to the Laplace problem. The method has been developed into a robust technique for especially modeling elasticity and acoustics [9] and [10]. In this study, the method was applied to the Laplace's equation for the exterior problems involving the evaluation of the capacitance between the concentric cylinders. The term "element" was used to mean the geometry and type of approximation to a given variable. On the other hand, the term "node" was used to define the element geometry or the variables involved in the problem.

In using the BEM on the interior Laplace problem, we noted that a different number of nodes is used for the variable approximations which may be linear, quadratic or otherwise. Some of these approximations 
like the linear and quadratic use global basis functions which are continuous over the boundary. Other approximations give a jump discontinuity which may not affect the accuracy of the method because of integration [2], [4], [11] and [12]. However, the use of spline approximation may be inevitable for the required continuity in the geometry [2].

Hence by applying the variable approximation equation (3) was approximated by equation (4):

$$
\left.\sum_{c=1}^{n}\left[\int_{s^{c}} G(x ; s) d s\right] \boldsymbol{V}_{c}-\left[\int_{\underline{S}_{c}} \frac{\partial G}{\partial n}(x ; s) d s\right] \boldsymbol{U}_{c}\right]=\left\{\begin{array}{c}
u(x) \text { if } x \in D \\
-0.5 u(x) \text { if } x \in S \\
0 \text { if } x \in E
\end{array}\right.
$$

The BEM has the corresponding exterior form in equation (5):

$$
\sum_{c=1}^{n}\left[\left[\int_{s} G(x ; s) d s\right] \mathcal{V}_{c}-\left[\int_{s} \frac{\partial G}{\partial n}(x ; s) d s\right] \boldsymbol{u}_{c}\right]=\left\{\begin{array}{c}
-u(x) \text { if } x \in D \\
-0.5 u(x) \text { if } x \in S \\
0 \text { if } x \in E
\end{array}\right.
$$

These variables were assumed to be constant on each element or node. For this case we chose one node per element, hence there are same number of nodes and elements. Therefore, obtaining $\underline{u}$ and $\underline{v}$ requires the application of the collocation method. Then, the variable point $\mathrm{x}$ is brought to each of the boundary nodes in turn [4]. The point $q_{r}$ was let to be the mid-point of element $r$. this led to the following equivalent equations:

$$
\begin{gathered}
\sum_{c=1}^{n}\left[\left[\int_{S^{c}} G\left(q_{r} ; s\right) d s\right] \boldsymbol{v}_{c}-\left[\int_{s} \frac{\partial G}{\partial n}\left(q_{r} ; s\right) d s\right] \boldsymbol{u}_{c}\right]=0.5 \boldsymbol{u}_{r} \\
\sum_{c=1}^{n}\left[\boldsymbol{L}_{r c} \boldsymbol{v}_{c}-M_{r c} \boldsymbol{u}_{c}\right]=0.5 \boldsymbol{u}_{r} \\
L_{r} v-M_{r} u=0.5 u_{r}
\end{gathered}
$$

where $r=1, \ldots, n$, and $n$ is the number of nodes. The exterior formulation simply negates the RHS of equation (6). It was noted that equation (6) can be written in matrix form as

$$
L \underline{v}-(M-0.5) \underline{u}=0
$$

for the exterior case [2]. For a Dirichlet problem where $\underline{u}$ is given, $\underline{v}$ can be found. We also noted on the other hand if it is a mixed problem, the resulting matrices have to be ordered and re-partitioned. The matrices $L_{r c}$ and $M_{r c}$ were resolved using the equations below for curved elements.

$$
L_{r c}=\int_{\bar{S}} G\left(q_{r} ; s\right) d s \text { and } \mathbf{M}_{\mathrm{rc}}=\int_{\bar{S}} \frac{\partial G}{\partial n}\left(q_{r} ; s\right) d s
$$


To resolve these integrals, we assumed the availability of the coordinates of the collocation point $q_{r}$ and the end points a, b. The diagonal entries arise when $q_{r}$ coincides with the element node. This accomplished the first step, because at this stage $\underline{v}$ could be calculated for a Dirichlet problem [2]. On the other hand, $\underline{u}$ at a general point could be found by

$$
\text { ü } \quad M \underline{x} t \quad L(x) \underline{v}
$$

Resolving the matrix and integral forms for the curved elements can be found in the findings of this manuscript.

\subsection{The Capacitance $(C)$}

The capacitance is the ratio of the charge on either plate $Q$ (charge held by the conductor) to the potential difference, $V$ between the plates. It is determined by the area/distance between the plates, and the nature of the dielectric.

$$
\begin{gathered}
C=\frac{Q}{V} \\
V=\frac{1}{4 \pi \varepsilon_{o}} \int \frac{\sigma d S}{r}
\end{gathered}
$$

$d S$ is an infinitesimal element of area, $r$ is the length from $d S$ to a fixed point $M$ within the plate. Using this method, the self-capacitance of a conducting sphere of radius $R$ is $C=4 \pi \varepsilon_{o} R$ [13].

This study was limited to the case of two conducting plates of arbitrary and different plate shapes. Ideally, the plates in this study are of different shapes which is a rear case. The usual cases that are normally handled are the Coaxial cable (Cylindrical and Spherical) and the Concentric cylinder (Spherical) as illustrated in Fig. 1 [13], [14]:

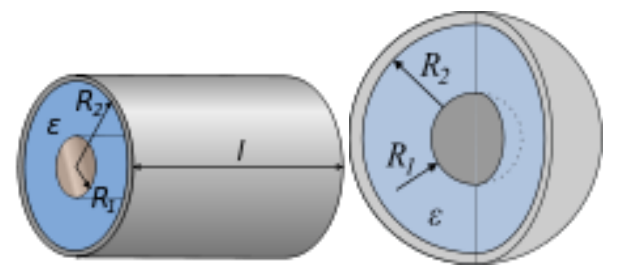

Fig. 1. Coaxial cable and the Concentric cylinder.

Capacitance also refers to the ability of a body to collect and store an electric charge. The body's shape can vary but curvatures can bring out any required shape for the plates. So curved elements are vital when combined with the BEM in managing such circumstances. A capacitor is a passive two-terminal electrical component that stores electrical energy in an electric field [13], [14].

The capacitance is a function only of the geometry of the design (e.g. area of the plates and the distance between them) and the permittivity of the dielectric material between the plates of the capacitor. For many dielectric materials, the permittivity and thus the capacitance, is independent of the potential difference between the conductors and the total charge on them [13]-[15]. 


\section{Methodology}

This study involved a review of the BEM. The Dirac-Delta and the Green's functions were also briefly reviewed in relation to the Laplace problem in the finite space.

The Laplace equation with its boundary integral formulation was done and the BEM applied. The algebra behind the elements was analyzed with more emphasis put on the curved elements to suit the approximation of the boundary for the different plates. Finally, three model problems were tested for analysis of the BEM and curved elements in relation to solving the Laplace problem. Several MATLAB programs were used in the analysis section. They included among others:

Beextcp. $m$, which uses the BEM for solving the Laplace's equation in the exterior of the Ellipse for a Dirichlet type problem. The same program was used in computing the capacitance between the concentric cylinders. The element data for this program and Beextc. $m$ is generated by a file eltsp. $m$ which requires only the number of nodes and ordinates. The values of the problem variable at the element mid-points i.e. the $\underline{u}$ data, is calculated from the exact solution which is calculated from exact solution which is calculated in a function sub-program ansep. $m$ (this returns the analytic value of a function at a sequence of points $\left(x_{1}, y_{1}\right) \ldots\left(x_{n}, y_{n}\right)$. [4]

Beextc. $m$, which uses the BEM for solving the Laplace's equation in the exterior of the unit circle for a Dirichlet type problem (Beextcp5. $m$ on the other hand uses a circle of radius 0.4 ). The element data, is calculated from the exact solution which is calculated in a function sub-program anse $1 . \mathrm{m}$. The function Eltsp $5 . m$ discretizes the boundary of the circle or radius 0.4 and stores such coordinates. [4]

\section{Findings}

\subsection{Computation of the Matrix Terms for Curved Elements}

The computation of the terms for the matrices $L_{r c}$ and $M_{r c}$ already considered in the previous section was done [4]. A curved boundary element, as an arc of a circle, $\mathrm{AB}$ was considered. The integrals $\mathrm{M}$ and $L$ were evaluated by mapping the curved element on to $[-1,1]$.

Assuming the availability of the end points $\mathrm{a}$ and $\mathrm{b}$ and the coordinates of the collocation point $q_{r}$, the computation of the matrix terms could be done [1], [4].

Equations (10) were simplified to:

$$
\begin{gathered}
M_{r c}=-\frac{l_{c}}{4 \pi} \int_{-1}^{1} \frac{(\underline{c}+\underline{p}) \cdot \underline{n} d t}{\lambda \sin ^{2}\left(\frac{\left.\alpha^{e} t\right)}{2}\right)+\cos \left(\frac{\left.\alpha^{e} t\right)}{2}\right)+\gamma} \\
L_{r c}=-\frac{l_{c}}{8 \pi} \int_{-1}^{1} \ln \left(\lambda \sin ^{2}\left(\frac{\left.\alpha^{e} t\right)}{2}\right)+\cos \left(\frac{\left.\alpha^{e} t\right)}{2}\right)+\gamma\right)
\end{gathered}
$$

The integrals in equations (10) were evaluated using the trapezoidal rule with a varying number of ordinates. The number of ordinates is denoted no in the computer programTrapez.m. However the diagonal elements given by the integrals $M_{r r}$ and $L_{r r}$ required a different approach because singularity [4]. The following work simplifies the terms for the singularity [16]-[20]. For the integral $M_{r r}$ the vectors $r$ and $n$ are orthogonal and therefore it is zero. So when 


$$
q_{c}=q_{r}, r(t)=\underline{p}=2 \rho \sin \left(\frac{\left.\alpha^{e} t\right)}{2}\right)
$$

Therefore the integral $L_{r r}$ was found to have a log type singularity and consequently needed the Gaussian quadrature approach for the improper integrals. The integral has a vertical asymptote [19] and $[21]$ on $[-1,1]$. In this approach we isolated the singularity by writing the integral as

$$
\int_{-1}^{1} \ln \left(\rho \alpha^{e} t\right) d t=\left(\int_{-1}^{-0.5}+\int_{-0.5}^{0}+\int_{0}^{0.5}+\int_{0.5}^{1}\right) \ln \left(\rho \alpha^{e} t\right) d t
$$

So that

$$
\frac{-l_{c}}{4 \pi} \int_{-1}^{1} \ln \left(\frac{\alpha^{e} t}{2}\right) d t=\frac{-l_{c}}{4 \pi}\left(\ln \left(\frac{l_{r}}{2}\right)-2\right) r=1,2, \ldots, n
$$

where $r=c$ and $\rho \alpha^{e}=\frac{l_{c}}{2}$

\subsection{Model Problem}

This problem from electro-statics required us to find the capacitance between two concentric cylinders [4], the elliptic inside the circular one as illustrated in Fig. 2 . The circle has radius $\mathrm{R}=0.4 \mathrm{~m}$, the ellipse with radii $0.2 \mathrm{~m}$, and $0.1 \mathrm{~m}$. Two boundary conditions were given: $u\left(\boldsymbol{S}_{e}\right)=0$ and $u\left(\boldsymbol{S}_{c}\right)=1$. Various methods were used though the analysis focused on the use of the BEM:

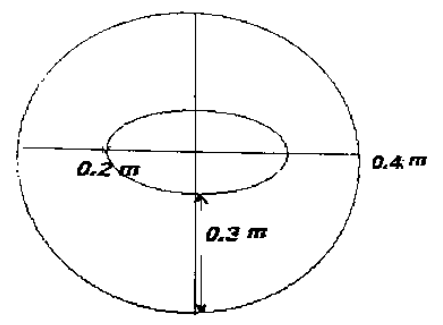

Fig. 2. The concentric cylinders.

Method I: We first of all used the ordinary method to compute the capacitance, without considering the boundary conditions. Let $+Q$ be the charge given to the ellipse (Inner plate). If the circle is assumed earthed, with air between them, then the induced charge on the circle (outer plate) is $-Q$, with $r_{1}$ and $R$ as the radius of the inner plate and the outer plate respectively. The potential of the ellipse, say $V_{e}$ is equal to the potential due to $+Q$ plus the potential due to $-Q$ as illustrated in equation (13).

$$
V_{e}=\frac{+Q}{4 \pi \varepsilon_{0} r_{1}}+\frac{-Q}{4 \pi \varepsilon_{0} r_{1}}
$$

The potential of the circle is Zero since it is assumed earthed. The BEM may help in this problem of the 
capacitance for different plates. Therefore, in using the ordinary computational method, we could only assume that the ellipse was also a sphere/circle with an average radius $r_{1}=0.15 \mathrm{~m}$ in order to establish the charge on the inner plate. Similarly, the potential of the circle $V_{c}$ is zero since the circle is assumed earthed [13]. The approximate capacitance $\mathrm{C}$, is given by

$$
\begin{gathered}
C=\frac{Q}{V}=\frac{4 \pi \varepsilon_{o} r_{1} R}{R-r_{1}} \\
\therefore \quad C=\frac{(4 \pi)(8.85)\left(10^{-12}\right)(.4)(.15)}{(.4-.15)}=26.6910 p F
\end{gathered}
$$

(The permittivity of free space if the charges are in a volume, $\boldsymbol{E}_{o}=8.854 \times 10^{-12}$ ) [13], [19].

Method II: We assumed the cylinders to be a cable of some length L. We neglected any supporting disks, and used Gauss' law. Thus

$$
\int_{S} \bar{E} \cdot d a=\frac{Q}{\mathcal{E}_{o}}
$$

where $\bar{E}$ is the electric field. The potential difference $\mathrm{V}$ is

$$
\begin{aligned}
V & =-\int_{S} \bar{E} d F=\frac{Q}{2 \pi L \mathcal{E}_{o}} \int_{r_{1}}^{R} \ln \left(\frac{R}{r_{1}}\right) \\
\therefore \quad C & =\frac{2 \pi \mathcal{E}_{o} L}{\ln \left(R / r_{1}\right)}
\end{aligned}
$$

The study also noted that the potential difference $V$ is a positive quantity because the inner cylinder is at a higher potential. It was further assumed that the length $\mathrm{L}$ is bigger than the radii $r_{1}$ and $R$. Therefore, no end effects were assumed.

For varying lengths, L, the capacitance is given in Table I below:

Table 1. The Capacitance with Varying Length [4]

\begin{tabular}{l|ccccc}
\hline $\begin{array}{l}\text { Length, L } \\
(\mathrm{m})\end{array}$ & 0.5 & 0.7 & 1.1 & 2.0 & 5.0 \\
\hline Capacitan & 28.332 & 39.665 & 62.331 & 113.328 & 283 \\
ce, C (pF) & & & & & .32 \\
& & & & & 1 \\
\hline
\end{tabular}

Method III: The justification of the BEM helps us to avoid the assumption about the inner plate. We apply the BEM to the problem by simply treating the situation as a connected domain and finally apply the exterior form to this Dirichlet problem.

Therefore, the capacitance could be given by the integral over either boundary of the normal derivative [15], [16]. Equation (10) for the potential difference could therefore be given by 


$$
\begin{aligned}
& V=-\int_{c} \bar{E} d F \quad x \in E \\
& =\frac{Q}{2 \pi L \varepsilon_{o}} \int_{c} V^{3}(x) d x
\end{aligned}
$$

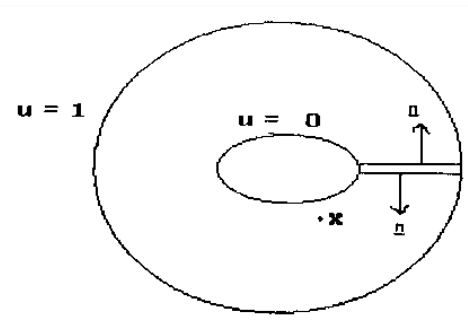

Fig. 3. The exterior form for this problem.

Equation (17) has varying values as $\mathrm{n}$ changes as shown in the Table 2 below:

Table 2. The Capacitance with Varying Nodes

\begin{tabular}{l|llllll}
\hline$n$ & 4 & 8 & 16 & 32 & 64 & 128 \\
\hline$V_{c}($ when $u=1)$ & 1.3429 & 0.7611 & 0.6503 & 0.7274 & 0.8288 & 0.8945 \\
$V_{e}($ when $u=0)$ & 0 & 0 & 0 & 0 & 0 & 0 \\
\hline
\end{tabular}

Thus the capacitance for some chosen length $L=0.5$ and $n=8$ can be given by:

$$
\begin{gathered}
C=\frac{2 \pi \mathcal{E}_{o} L}{V_{c}-V_{e}} \\
=\frac{2(3.14)(.5)(8.85)\left(10^{-12}\right)}{.7611} \\
=36.5116 p F
\end{gathered}
$$

Table 3 was used to illustrate and give varying $\mathbf{C}$ values as seen in the table below:

Table 3. The Capacitance with Varying Number of Nodes

\begin{tabular}{l|lll}
\hline Length, $L(\mathrm{~m})$ & \multicolumn{1}{c}{$n=8$} & \multicolumn{1}{c}{$n=64$} & $n=128$ \\
\hline & $\mathrm{C}(\mathrm{pF})$ & $\mathrm{C}(\mathrm{pF})$ & $\mathrm{C}(\mathrm{pF})$ \\
0.5 & 36.512 & 33.529 & 31.067 \\
0.7 & 51.116 & 46.941 & 43.496 \\
1.1 & 80.326 & 73.764 & 68.3463 \\
2.0 & 146.047 & 134.117 & 124.266 \\
5.0 & 365.116 & 335.292 & 310.665 \\
\hline
\end{tabular}

\section{Discussion}

This was conformable with the fact that the more the boundary of the ellipse is discretized, the better it is approximated and the better the accuracy of the solution. 
The same ellipse was made to be the inner cylinder and the circle of radius 0.4 , the outer cylinder. It is hardly possible to find the capacitance if the charges on the inner and outer cylinders is different. The BEM and curved elements can cater for this through increasing the number of elements on the ellipse boundary. Therefore, the capacitance that follows equation (14) could be compared to that in table III for the smallest length considered. The capacitance in table I was observed to be better because the cylinder was assumed some length $L$. As this length increased, the capacitance was increasing as well because it is proportional to its length.

Using the numerical analysis techniques, we observed that this was a Dirichlet problem [21], [22] which could also be handled using the BEM for curved elements. Such elements can be used to avoid the assumption about the radius of the ellipse. This capacitance was seen to be proportional to the integral over either boundary of $\boldsymbol{v}$, which could be found after applying the BEM using the given $\boldsymbol{u}$. So $n$ was varied on the circle and ellipse to give some of the results in Table 3. In fact when $n=256, C=29.8839 p F$, and Table IV gives varying length and respective proportional capacitance.

Table 4. The Capacitance When $N=256$ with Varying Length, $\mathrm{L}$

\begin{tabular}{l|ccccc}
\hline $\begin{array}{l}\text { Length, } L \\
(m)\end{array}$ & 0.5 & 0.7 & 1.1 & 2.0 & 5.0 \\
\hline $\begin{array}{l}\text { Capacitanc } \\
\text { e, C (pF) }\end{array}$ & $\begin{array}{c}29.88 \\
4\end{array}$ & 41.837 & 65.745 & 119.535 & 298.8 \\
\end{tabular}

The above discussions show that curved elements and the BEM applied to a two-dimensional exterior Laplace problem were better than other methods. Moreover, better solutions are obtained if such elements are applied to cases that have curvatures like the potential and capacitance problems a discussed above. In fact, when straight elements are applied to such cases, they are either too much of approximations or they completely fail to work. For example, unless we use the curved elements, it becomes very difficult to establish the charge on the elliptic problem as the problem above.

\section{Future Work}

There are various areas that the study may suggest for future work. However, only two were considered: The plates considered in this study were the circular and elliptic which are directly related to curved elements. Future work from this may be considering a mixture of both the curved and straight element types. The second case may be considering more than 2D cases in boundary discretization procedure and model problem beginning with the 3D cases.

\section{Acknowledgment}

This work was made possible through project funding by Busitema University where we are employed.

\section{References}

[1] Wrobel, L. C, \& Aliabad, M. H. (2002). The Boundary Element Methods in Engineering. McGraw-Hill College.

[2] Semwogerere, T. (2015). An analysis of the curved elements to a 2D laplace problem using the BEM. Journal of Engineering Mathematics and Physics, 5(3), 167-176.

[3] Rjasanow, S., \& Weggler, L. (2013). Accelerated high order BEM for maxwell problems. Journal of Computational Mechanics, 51, 431-441.

[4] Semwogerere, T, (2016). The formulation and discretization analysis using the BEM. Proceedings of the Conference Proceedings with ISBN 978-1-63248-069-9 and Archived in Seek Digital Library. 
[5] Henwood, D. J., \& Kirkup, S. M. (1994). An empirical analysis of the boundary element method applied to Laplace'S equation. J. App. Mathematical Modeling, 2, 32-36.

[6] Curtis, F. G., \& Wheatly, O. P. (1994). Applied Numerical Analysis, $5^{\text {th }}$ ed. Wadsworth Publishing Company, Belmont, California.

[7] Wu, H. J., Liu, Y. J., \& Jiang, W. K. (2013). A low frequency fast multiple boundary element method based on analytical integration of the hyper singular integral for 3d acoustic problems. J. Engineering Analysis with Boundary Elements, 37, 309-318.

[8] Zhu, S., Satravaha, P., \& Lu, X. (1994). Solving linear diffusion equations with the dual reciprocity method in Laplace space. J. Engineering Analysis with Boundary Elements, 13, 309-318.

[9] Bremer, J., \& Gimbutas, Z. (2012). A Nystrom method for weakly singular integral operators on surfaces. J. Computational physics, 231, 4885-4903.

[10] Gao, X. W., \& Davies, T. G. (2002). Boundary Element Programming in Mechanics. Cambridge University Press (ISBN: 052177359-8).

[11] Gao, X. W. (2010). An effective method for numerical evaluation of general 2D and 3D high order singular boundary integrals. J. Computer Methods in Applied Mechanics and Engineering, 199, 2856-2864.

[12] Tipler, P., \& Mosca, G. (2004). Physics for Scientists and Engineers (5th ed.). Macmillan.

[13] Massarini, A., \& Kazimierczuk, M. K. (1997). Self-capacitance of inductors. IEEE Transactions on Power Electronics, 12(4), 671-676.

[14] Jackson, J. D. (2000). Charge density on thin straight wire, revisited. Am. J. Phys., 68(9), 789-799.

[15] Carley, M. (2007). Numerical quadrature's for singular and hyper singular integrals in boundary element methods. J. Scientific Computing, 29, 1207-1216.

[16] Graglia. R. D., \& Lombardi, G. (2008). Machine precision evaluation of singular and nearly singular potential integrals by use of gauss quadrature formulas for rational functions. IEEE Transactions on Antennas and Propagation, 56, 981-998.

[17] Kolm, P., \& Rokhlin, V. (2001). Numerical Quadratures for singular and hypersingular integrals. J. Computers and Mathematics with Applications, 41, 327-352.

[18] Khayat, M. A., \& Wilton, D. R. (2005). Numerical evaluation of singular and near-singular potential integrals. IEEE Transactions on Antennas and Propagation, 53, 3180-3190.

[19] Fata, S. N. (2009). Explicit expressions for 3D boundary integrals in potential theory. J. Numerical Methods in Engineering, 78, 32-47.

[20] Zheng, R., Coleman, C. J., \& Phan-Thien, N. A boundary element approach for non-homogeneous potential problems. J. Computational Mechanics, 7, 279-288.

[21] Henwood. D. J. (1997). The boundary element method applied to Laplace's equation. J. Audio Eng. Soc., 6, 1-19

[22] Rjasanow, S., \& Weggler. L. (2013). Accelerated high order BEM for Maxwell problems. J. Computational Mechanics, 51, 431-441.

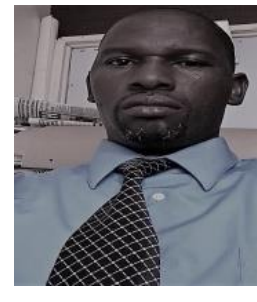

Twaibu Semwogerere was born in Uganda in August, 1973, and holds a bachelor of science education degree (1995) (mathematics), the master of science degree (2002) (applied mathematics) of Makerere University, Kampala, Uganda. He has a work experience of over 22 years in colleges and university teaching. Published several papers, the previous one being: "Community Policing About Accidental Risks and Road Usage in Uganda." in the proceedings of the 71st, International Conference on Tourism, Transport, 
and Logistics (ICTTL) held in June, 2017 in Kuala Lumpur, Malaysia. He is currently a Senior Lecturer in the Faculty of Engineering, Busitema University.

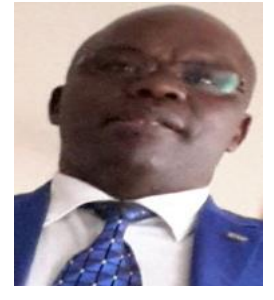

Richard Awichi holds a bachelor of science with education degree from Makerere University, Kampala Uganda with a major in mathematics. He completed this degree in 1994. He later enrolled for a master's degree in Mathematics from Makerere University which he completed in 2002. He specialized in mathematical statistics. He obtained a PhD in mathematical statistics from Johannes Kepler University, Linz, Austria in 2015. He has taught mathematics in universities in Uganda since 2000. His recent publication (in Jan 2018) was on the topic: "On Spatial Dependence in Multivariate Singular Spectrum Analysis" in the Journal of Mathematics and Statistical Sciences. He is currently the head of Mathematics Department in the Faculty of Science, Busitema University.

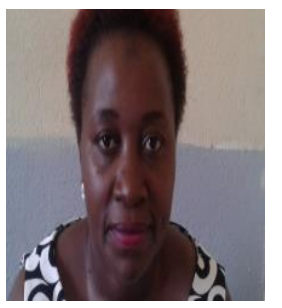

Hasifa Nampala obtained a bachelor of science with education degree in 1998 from Makerere University, with teaching subjects as mathematics and physics. She later graduated with MSc in mathematical modelling, with a bias in financial mathematics from the University of Dar-es-Salam, Tanzania and Lappeenranta University of Technology, Finland in 2010. She obtained her PhD in mathematics from Makerere University in 2016 and her topic was 'Modelling hepatotoxicity and antiretroviral therapeutic effects in HIV-HBV coinfection". Her recent publication is "modelling effective antiretroviral therapy that inhabits HIV production in the liver", in the journal of antivirals and antiretrovirals. A manuscript has been accepted by mathematical biosciences in February 2018 on "Modelling hepatotoxicity of antiretroviral therapy in HIV/HBV coinfection". Hasifa started teaching mathematics in 1998 and is currently a lecturer of mathematics at Kyambogo University. 